\title{
Complex Scenarios with Competing Factors - A Conception Paper Applied to the COVID-19 Case
}

Mauricio Pazini Brandão ( $\sim$ pazinibrandao@gmail.com )

Ministry of Science, Technology, and Innovations https://orcid.org/0000-0001-5445-7354

\section{Research Article}

Keywords: Energy Principle, Resources Allocation, Decision-Making, Macroeconomics, Epidemics, COVID19

Posted Date: August 3rd, 2020

DOl: https://doi.org/10.21203/rs.3.rs-51369/v1

License: (9) This work is licensed under a Creative Commons Attribution 4.0 International License. Read Full License

Version of Record: A version of this preprint was published at Nonlinear Dynamics on April 21st, 2021. See the published version at https://doi.org/10.1007/s11071-021-06361-y. 


\title{
Complex Scenarios with Competing Factors
}

\section{A Conception Paper Applied to the COVID-19 Case}

\author{
Mauricio Pazini Brandão \\ Ministry of Science, Technology, and Innovations \\ pazinibrandao@gmail.com +55 12 99718-8599
}

Keywords: Energy Principle, Resources Allocation, Decision-Making, Macroeconomics, Epidemics, COVID-19

\begin{abstract}
A model to analyze complex systems facing threats with competing factors has been introduced. The Principle of Energy, in integral form, is used to conceive a theory in which competing factors dispute available resources to minimize undesirable outcomes. The general result indicates that the minimum response is obtained by a combination of the factors weighted by their corresponding criticalities. The theory has been applied to the case of the COVID-19 pandemic with two competing factors: Health and Economy. As result, to minimize the grand total number of deaths, the best recommendation is to balance the emphasis on both factors. The model can be generalized even further and may evolve from a qualitative to a quantitative status. In this evolution, it may allow for computational simulations and comparisons with field statistics for validation and forecasting. As such, this approach may become a useful tool for decision-making regarding resources allocations in order to reduce guessing in scenarios full of uncertainties.
\end{abstract}




\section{1 - THE SCENARIO}

No matter how complex a situation might be, there might be a way of watching it from a perspective characterized by simplicity. In this paper we introduce an approach to treat a general problem with a simple yet powerful tool inspired in Nature.

Let us imagine some sort of domain (a country, a planet, a solar system, or even a galaxy) where, all of a sudden, there appears an internal threat. This threat produces undesirable results that will be called here outcomes.

Let us emphasize that the threat is internal to the domain. Therefore, the domain should be regarded as a closed system. No external inputs are considered in the problem. The threat imposes to the domain a certain load. The domain has to accept this load and respond to it with outcomes. Since the threat is considered a bad thing, it is desirable that the outcomes are kept at minimum levels.

To fight the threat, the creatures of the domain have some means of support that will be called here resources. The resources may be employed to combat the threat in different forms that will be called here factors. The factors compete among themselves for the resources in a non-collaborative form. Therefore, each resource may be used to deal with one factor or another, but not used simultaneously by more than one factor.

The outcomes are countable numbers. The factors are countable and discrete numbers. They are different forms of allocating the resources to mitigate the threat. On the other hand, the resources may be either countable or uncountable. They may be either limited or unlimited. In the case of being limited, they pose definitive restrictions that the solution to the problem must observe.

In summary, the threat produces undesirable outcomes. The domain creatures want them to be minimum. To reach this goal, the creatures use resources at their own will, but they wish to have some sort or rational guidelines to make sure that the resources are allocated for best cost/benefit ratio. The scenario is complex in nature. The solution sought is a rational use of the available resources to minimize the outcomes.

\section{2 - THE PRINCIPLE OF ENERGY}

In our planet - and perhaps in the whole universe - it is known that energy is a quadratic expression of system variables. Once subjected to loads, system internal stresses are negotiated so that its equilibrium is achieved when the energy absorbed by the system reaches a minimum. This is the inspiration we use here to seek a solution for the proposed threat-outcome problem. It is simply Nature acting at its own manner.

Let us consider a closed system (domain). Internally to this system, there appears a load (threat). The load acts within the system doing some work. The system absorbs the load, distributes the resulting stresses throughout itself, and provides an answer (outcome). It is wanted to establish some sort of correlation between the load and the answer and, if possible, to determine the answer. 
If one considers all the forms of energy and work involved in this case, it is possible to add up all the contributions in an integral objective function I. The Principle of Energyexpressed in terms of Variational Calculus (Gelfand and Fomin [1], Goldstine [2], and Fergusson [3]) - says that the first variation of I is null, i.e.

$$
\delta \mathrm{I}=0
$$

Therefore, if one is capable of obtaining I as a function of all system variables, the problem can be solved by minimizing I. Nature is such that this mathematical process leads to a minimum because it is known that the second variation of I is positive, i.e.

$$
\delta^{2} \mathrm{I}>0
$$

This is the mathematical basis used to develop a similar solution to the threat-outcome problem. We imply that it is possible to write an objective function in integral form for the problem and that it can be minimized in terms of the system variables. To show how this works, let us discuss the simplest of the cases and make generalizations afterwards. We will keep the Mathematics in the text as simple as possible, just for the purpose of discussion. The relevant details and extensions appear in the Annex.

\section{3 - THE BASELINE PROBLEM}

Let us suppose, for the sake of simplicity, that we have a scenario where the number of factors is two and that the amount of resources is unlimited. Under these hypotheses, the problem can be developed on a plane for easy visualization.

Figure 1 depicts the baseline solution. The threat is absorbed by the entire system in uniform fashion because there is no competition between the two factors. In mathematical terms, the two factors are independent or, in practical terms, Factor 1 does not feel the existence of Factor 2 and vice-versa.

This is the limiting case in which

$$
\delta^{2} \mathrm{I}=0
$$

The value of the uniform outcome depends on the amplitude of the threat and on the system internal capability of absorbing this amplitude, something that in Engineering is called the system impedance or stiffness. Let us denote this baseline outcome by $O_{b}$. 


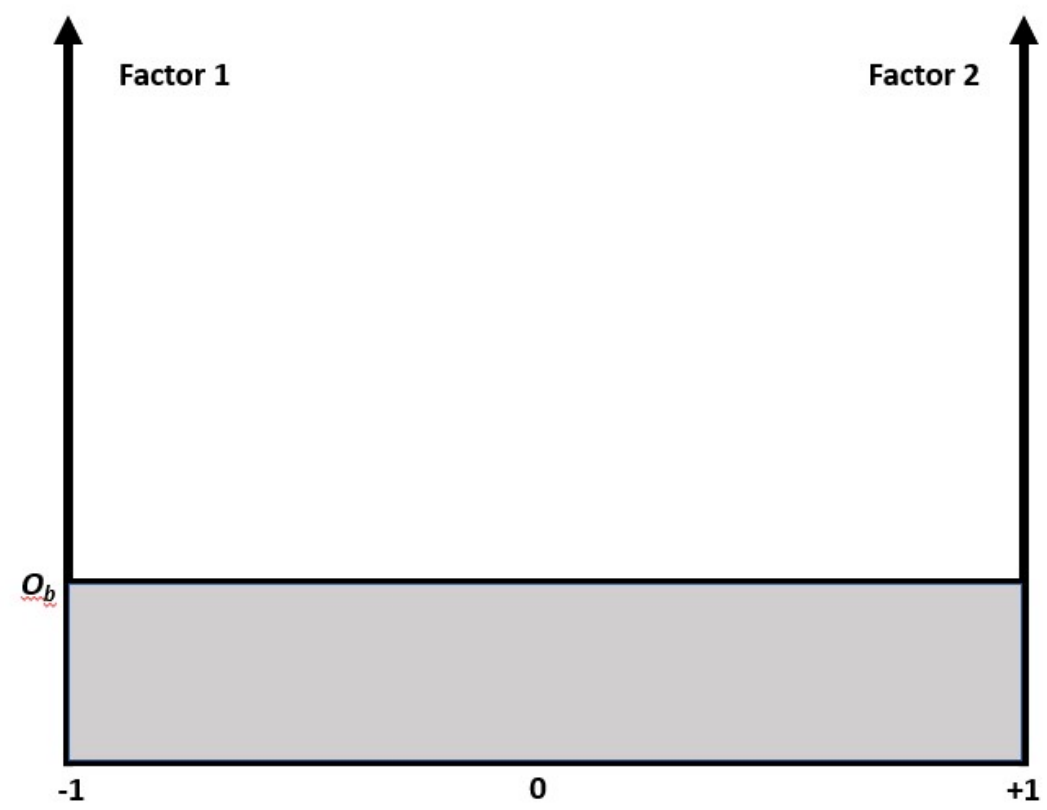

Figure 1 - The baseline unrestricted problem.

In Figure 1, the value -1 in the abscissas represents a condition where there is $0 \%$ weighting of Factor 2 and $100 \%$ weighting of Factor 1 . Similarly, the value +1 represents the counterpart situation where there is $0 \%$ weighting of Factor 1 and $100 \%$ weighting of Factor 2. Exactly in the middle, the value 0 in the abscissas displays the condition where both factors have the same weighting, that is $50 \%$ for each one.

The baseline outcome $O_{b}$ represents the accepted minimum response of the system to the threat. This outcome is indifferent to the decision of facing the threat by giving more emphasis to one factor or to the other because the resources are unlimited.

Now we pose a crucial question: what happens if one considers that one or more of the resources is limited? In this case, the factors start competing for the resources. In other words, one factor becomes aware of the existence of the other. A well-educated guess comes to stage: the once constant straight line shown in Figure 1 now becomes a curve with positive concavity, i.e., with positive second variation.

The reason for this behavior is not difficult to understand. If Factor 1 grabs more resources than Factor 2, the performance of both changes. Factor 1 will continue to display results not very different from those obtained in the unrestrained baseline case, whereas Factor 2's performance will be poorer. The sum of the performance of both factors will be such as, for the same system impedance or stiffness, to increase the level of the undesirable outcomes. Therefore, the resulting curve goes above the $O_{b}$ level for any combination of limited resources distribution. 
Figure 2 depicts the perfectly symmetrical case where both Factors have similar competences in gathering resources for themselves and similar performances in applying them to give the threat a combat. Besides, we consider here that they both yield the same maximum outcome $O_{m}$ when they obtain $100 \%$ of all available resources.

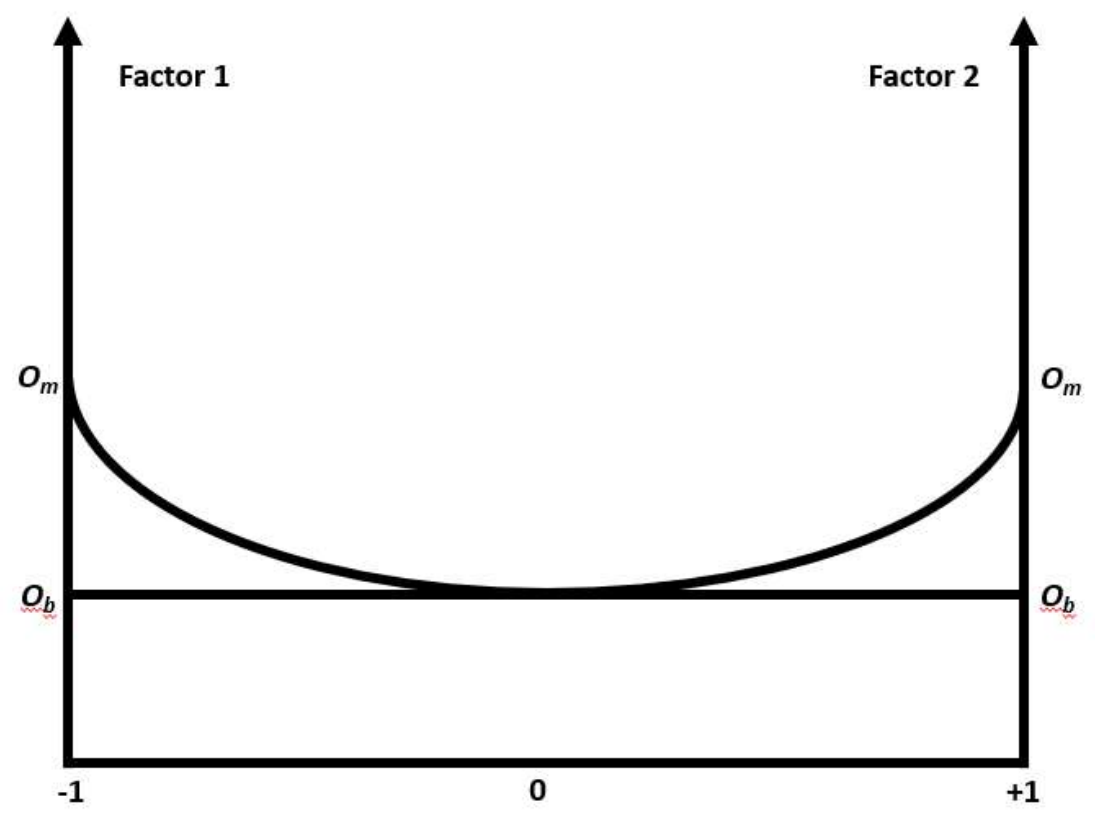

Figure 2 - The perfectly symmetrical competing problem.

In Figure 2, the abscissa -1 represents the condition where it is given $100 \%$ emphasis to Factor 1 and $0 \%$ emphasis to Factor 2. Thus, the difference between the outcomes $O_{m}$ and $O_{b}$ is mainly due to underemphasizing Factor 2 . Similar considerations - like in a mirror - are implied for the condition given in abscissa +1 .

In this case, there is no doubt that the minimum outcome happens when equal attention is given to both Factors. For the sake of simplicity, we consider here that the $50 \%-50 \%$ split condition yields as minimum outcome the baseline value $O_{b}$, but this is not an obligatory result. In fact, it is an optimistic result. Most probably, the existence of competition for resources between the two Factors will produce a minimum outcome value that is higher than $O_{b}$.

Figure 3 shows an asymmetrical case of competition between the two Factors. In this Figure, the maximum outcome $O_{m 1}$ of Factor 1 is bigger than the maximum outcome $O_{m 2}$ of Factor 2. Besides, the minimum of the curve happens between the midpoint 0 and the +1 point. This means that the impact of Factor 2 on the maximum outcome $O_{m 1}$ is bigger than the impact of Factor 1 on the maximum outcome $O_{m 2}$. In this case, Factor 2 is identified as being more critical than Factor 1. 


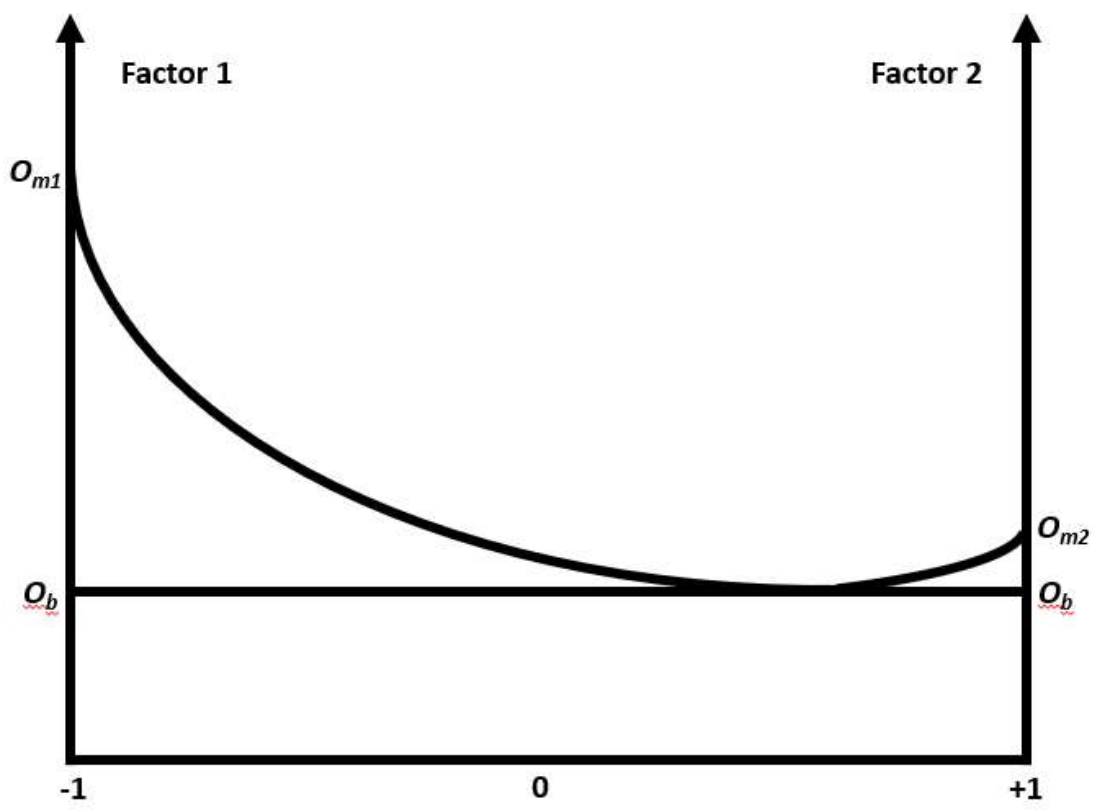

Figure 3 - An asymmetrical competing problem.

The idea in the asymmetric case is altruistic, in the sense that the more resources you bring to your factor - the egotistic way, the more bad results you collect that come from neglecting the other factor. In the case shown in Figure 3, Factor 1 is less critical than Factor 2 because placing emphasis on Factor 1 yields greater outcomes than placing emphasis on Factor 2. Therefore, in order to minimize the outcome, it makes more sense to invest in Factor 2 than in Factor 1.

Figure 4 shows the limiting asymmetrical case where the minimum is at the +1 point. The totality of the criticality of the system is placed on Factor 2. In this case, if all resources are invested on Factor 2, the outcome is, for the sake of simplicity, the same of the baseline case $\left(O_{b}\right)$. On the other hand, if all resources are invested on Factor 1 , the maximum outcome $O_{m}$ is bigger than the baseline value $O_{b}$. In other words, the final outcome is inherited from the criticality of Factor 2, from the fact of Factor 2 having been deprived from needed critical resources.

What must be understood in this threat-outcome problem is that the final objective is to obtain the minimum outcome. For such condition, it is absolutely necessary to discover which Factor is the critical one and to invest more on it. Since the case depicted in Figure 4 is an ideal limit, it is worth discovering in a real problem where the minimum point is, like in the cases shown in Figures 2 or 3.

Within the conception presented here, let us now discuss qualitatively its application to the WHO SARS-COV-2 (COVID-19) case. 


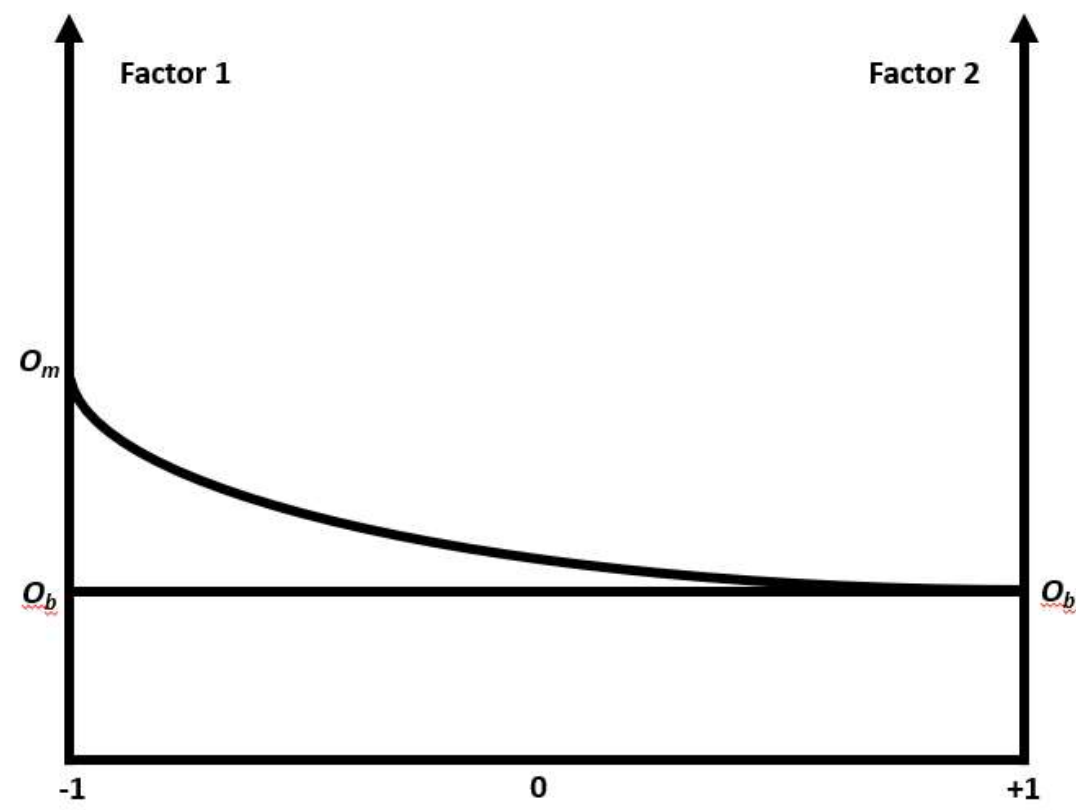

Figure 4-A limiting asymmetrical competing problem.

\section{4 - APPLICATION TO THE COVID-19 CASE}

\section{1 - The Scenario without Competition}

Let us consider the hypothetical situation in which a country is invaded by a virus outbreak, subsequently elevated to pandemic status. This is an undesirable threat. People fear its outcomes because there is no vaccine, but there is no way out. The threat must be faced with all the country's resources. Fortunately, the country possesses a set of means for mitigating the threat. People want to use them well, but they wonder which is the best way of doing it.

Let us start the analysis in search for a possible answer to this problem. Let us consider the baseline solution, that's to say, the one in which the country has no vaccine, but has all the remainder needed resources, and better yet, they are unlimited.

In this ideal scenario, there is little modification in the country's modus vivendi, for the country's resources are plentiful. To name a few:

1. Infrastructure - hospitals, health centers, ambulatories, clinics, all well installed, geographically well distributed, and with sufficient beds either in infirmary or ICU;

2. Logistics - sufficient means of transportation for people and delivery of supplies in order to make all subsystems to function flawlessly;

3. Personnel - doctors, nurses, and attendants, all well-educated, prepared, and trained, in enough quantity to offer prompt services with quality; 
4. Equipment - adequate equipment and working supplies provided by national industries as needed, in the short term, at fair prices;

5. Treatment - effective drugs and therapies with approved protocols for the clinical care of the patients, despite the novelty of the disease;

6. Budget - sufficient money to pay for all services at all levels of the Executive Branch, federal, state, and county;

7. Policies - public authorities of all government branches working in harmony, each in its specific role, supporting each other;

8. Living conditions - enough food, plenty of energy, adequate sanitation, and everything else to overcome the transitions imposed by the threat;

9. Guidance to the population - orientation on how people should behave to reduce contamination, decrease chances of getting sick, seek proper attendance, etc; and

10. People's will - people and institution's intentions to fight the threat, to minimize the number of deaths, and to prepare society for the future.

The above is a non-exhaustive list of resource groups that may be used to generate mathematical model parts and identification of variables for the problem. They provide a taxonomy to keep in reference: infrastructure, logistics, personnel, equipment, treatment, money, political support, living conditions, orientation, and people's will.

In this ideal scenario, until vaccines come out, loss of lives due to the pandemic have to be accepted - and mourned - by society as an unfortunate but natural phenomenon. Besides the health-related orientations, there is no need for any other measures and everyone's lives go on as normal as possible. Furthermore, there is little to question from the economic and from the psychosocial standpoints.

The previous list of resources can be cast, for the sake of systematization, in two factors: Health $\left(\alpha_{1}\right)$ and Economy $\left(\alpha_{2}\right)$. It can be realized that some resources have very strong relation to Factor 1; others, to Factor 2. However, one can also realize that some of them have relational links to both factors, which means that they are definitively coupled.

In the hypothetical scenario, if we create a graph of the total number of deaths due to the pandemic on the vertical axis versus prioritizing the Health and Economy factors in the horizontal axis, the result is a horizontal line going from the $100 \%$ Health / $0 \%$ Economy to the $0 \%$ Health / $100 \%$ Economy points. In other words, the graph is exactly like the horizontal line presented in Figure 1, extending from the -1 till the +1 point.

In the ideal scenario, with plentiful of resources, society, seen as a system, is not very much stressed and the number of deaths $O_{b}$ does not make, unfortunately, newspapers headlines in big letters. In other words, there is no competition between the two factors and the death toll is accepted as a normal phenomenon.

In order to lower the number of deaths $O_{b}$, the general recommendation is to make investments in Education, Science, Technology, and Innovation (EST\&I). These investments have structuring capabilities, in the sense that they improve the performance of all groups of resources. This is equivalent to increasing the society impedance or stiffness as a whole and, thus, to lowering the system outcomes. 


\section{2 - The Scenario with Competition}

The perfect scenario described in the previous section begins to change when one realizes that resources are finite and that some sort of restrictions should be applied, either on Health $\left(\alpha_{1}\right)$ or on Economy $\left(\alpha_{2}\right)$. Modeling on the Health Factor seems to be simpler, based on more tangible indicators, variables and restrictions. Modeling on the Economy Factor seems to be more difficult, as it is based on many intangible indicators.

In the real world, infrastructure is lacking, logistics has flaws, there is not enough personnel, equipment and supplies become expensive, there are no medicines or acknowledged protocols for treatment of a new disease, and budget misses timing and magnitude. In addition, political actions are confusing and non-collaborative, living conditions become poorer, people have no access to proper and opportune guidance, and people's will fades, leading in some cases to depression or even to suicide.

All these elements can be individually modeled in quadratic forms and these forms can be weighted and integrated to compose factors that are components of the integral objective function I to be minimized.

For both factors, there are several elements (resources, variables, and restrictions) to consider in the model. Let us take, for instance, the number of ICU beds as an element. In countries where there are plenty of them, they may be regarded as resources and, perhaps, variables. In countries where they are lacking, they should be regarded as restrictions. The same perception stands for lung respirators and respiration tents.

By placing strong emphasis on the restrictions, some authorities have used the collapse of the country's Health System to justify rigid social isolation measures. However, these isolation measures harm the country's economics.

Let us consider as general economic losses the closure of companies, the rising of unemployment, the starvation, and resulting deaths. Let us add the depression caused by isolation, the conflicts resulting in fights and deaths. Let us also consider the psychological effects on people due to isolation, some leading to suicide. And the list continues. Therefore, as much as there are deaths due to the virus because of health problems, there are deaths due to the virus because of economic reasons.

Without having developed very detailed models of the whole problem, including all possible elements from both factors, authorities have only partial, fuzzy, or even meaningless knowledge to base their decisions upon. The point is that, as of now, it is unclear which of the two factors is the critical one in the sense discussed in this paper. Until we have enough analyses, results, and field evidences to clarify this issue, the wisest position to take is to consider that we have in hand a symmetric case.

Anyway, it makes reasonable sense to consider that in the real case there is an optimum point to minimize the number of deaths, like those points appearing in Figures 2 or 3. For the sake of equilibrium and justice, considering both factors equally critical, here we will take the symmetric case (Figure 2) to obtain a qualitative closure of this study and to reach some conclusions. 


\section{3 - A Mathematical Application}

Let us consider as the problem objective function a general and complete quadratic form of factors $\alpha_{1}$ (Health) and $\alpha_{2}$ (Economy) as follows:

$$
\mathrm{I}\left(\alpha_{1}, \alpha_{2}\right)=\mathrm{A} \alpha_{1}^{2}+\mathrm{B} \alpha_{2}^{2}+\mathrm{C} \alpha_{1} \alpha_{2}+\mathrm{D} \alpha_{1}+\mathrm{E} \alpha_{2}+\mathrm{F}
$$

Here, the coupling between the two factors appears explicitly on the $C, D$, and $E$ terms. We lose no generality if we normalize I by making $A=1$ and if we set the constant value $F=0$. In this case, we are left with the four coefficients $B, C, D$, and $E$ to determine.

The application of the Principle of Energy (Equation 1) to Equation 4 yields

$$
\delta I=\left(2 \alpha_{1}+C \alpha_{2}+D\right) \delta \alpha_{1}+\left(2 B \alpha_{2}+C \alpha_{1}+E\right) \delta \alpha_{2}=0
$$

Since the variations $\delta \alpha_{1}$ and $\delta \alpha_{1}$ are both different from zero in the general case, we obtain the following set of equations:

$$
\left[\begin{array}{cc}
2 & C \\
C & 2 B
\end{array}\right]\left\{\begin{array}{l}
\alpha_{1} \\
\alpha_{2}
\end{array}\right\}=\left\{\begin{array}{l}
-D \\
-E
\end{array}\right\}
$$

from where we get

$$
\alpha_{1}=\frac{2 B D-C E}{C^{2}-4 B} \quad \text { and } \quad \alpha_{2}=\frac{2 E-C D}{C^{2}-4 B}
$$

These are the values for $\alpha_{1}$ and $\alpha_{2}$ where the minimum of I occurs. By proper modeling of the factors according to the resources described in section 4.1, numbers can be assigned to the coefficients B, C, D, and E. From these assignments, we can determine the values of $\alpha_{1}$ and $\alpha_{2}$ and identify which of the two factors is the most critical one.

In order to obtain graphics like those displayed in Figures 1 to 4, we need to map the quadratic objective function (4) onto the linear domain $-1 \leq \sigma \leq+1$. For this purpose, we make the following linear transformations

$$
\alpha_{1}=\frac{1}{2}(1-\sigma) \quad \text { and } \quad \alpha_{2}=\frac{1}{2}(1+\sigma)
$$


With this linear transformation, factor $\alpha_{1}$ has the normalized value $1(100 \%)$ at $\sigma=-1$ and decreases to the value $0(0 \%)$ at $\sigma=+1$, whereas factor $\alpha_{2}$ has the value 0 at $\sigma=-1$ and increases to the value 1 at $\sigma=+1$. At the midpoint $\sigma=0$ both factors have the same value 0.5 (50\%). This linear transformation brings the quadratic form I displayed in Equation (4) to a new quadratic form $\mathrm{I}_{m}$ - subscript $m$ for mapping - such as

$$
I_{m}(\sigma)=1 / 4\left[A(1-\sigma)^{2}+B(1+\sigma)^{2}+C\left(1-\sigma^{2}\right)+2 D(1-\sigma)+2 E(1+\sigma)+4 F\right]
$$

Again, there is no loss of generality if we set $A=1$ and $F=0$. For the determination of the remainder coefficients $B, C, D$, and $E$, in the symmetric case discussed in section 3 , we apply the following boundary conditions which yield equal maxima $O_{m}$ at the extremities $\sigma=-1$ and $\sigma=+1$, and minimum $O_{b}$ at the midpoint $\sigma=0$ :

$$
\begin{aligned}
& \mathrm{I}_{m}(-1)=O_{m} \\
& \mathrm{I}_{m}(+1)=O_{m} \\
& \mathrm{I}_{m}(0)=O_{b} \\
& \mathrm{dI}_{m} / \mathrm{d} \sigma(0)=0
\end{aligned}
$$

After clearing the algebra, we obtain the following results for the coefficients of equation (9):

$$
\begin{aligned}
& \mathrm{A}=1 \\
& \mathrm{~B}=2 O_{m}-4 O_{b}-1 \\
& \mathrm{C}=-2 O_{m} \\
& \mathrm{D}=O_{m}-1 \\
& \mathrm{E}=4 O_{b}-O_{m}+1 \\
& \mathrm{~F}=0
\end{aligned}
$$

These results reproduce the curve presented in Figure 2 . Besides, they confirm that we obtain a minimum at the midpoint $\sigma=0$, provided $O_{m}>O_{b}$, i.e.,

$$
\mathrm{d}^{2} \mathrm{I}_{m} / \mathrm{d} \sigma^{2}(0)=1 / 2(\mathrm{~A}+\mathrm{B}-\mathrm{C})=2\left(O_{m}-O_{b}\right)>0
$$


In the limit where $O_{m}=O_{b}$, we obtain

$$
\mathrm{d}^{2} \mathrm{I}_{m} / \mathrm{d} \sigma^{2}(0)=1 / 2(\mathrm{~A}+\mathrm{B}-\mathrm{C})=2\left(O_{m}-O_{b}\right)=0
$$

which is the unrestricted baseline case discussed in section 3, presented in Equation (3), and displayed in Figure 1.

\section{4 - Discussion on Social Isolation}

Since still there is no vaccine yet for the COVID-19 virus, the solution now calls for herd immunity. Presently and understandably, in several countries, more emphasis is being given to the Health Factor than to the Economy Factor. Behind this implicit emphasis is the explicit model of social isolation. Health claims that, in order to save lives, social isolation should be horizontal, if not total lockdown. Some countries have, on the other hand, applied vertical isolation or even no isolation at all. The comparison between the two positions is inconclusive because it has been based on incomplete premises. One has first to model the lives lost due to economic effects and compare to the lives lost due to the virus and to other untreated diseases. And, so far, to our knowledge, there has been no studies performed with comprehensive and balanced approaches.

To apply horizontal social isolation or lockdown is equivalent to place $100 \%$ emphasis on the Health Factor. We have shown here that this is not the optimum solution, for it will raise the total number of deaths. The optimum $50 \%-50 \%$ (split) symmetric solution suggests an equivalence to the vertical isolation model. Therefore, in order to save lives, the conclusion is to increase the emphasis on the Economy Factor to reduce the total system stresses, to bring the system to an equilibrium, and to build a win-win condition. Equilibrium here means that the grand total stress of the system will be lower than it has been since the beginning of the pandemic in most of the countries.

The best solution, therefore, is not in a model of horizontal and radical social isolation nor in a model of total openness. Isolation should be applied to people who are in health conditions called at risk, with comorbidities. Although without definitive quantitative results at the moment, but with reasonable scientific evidence, it seems that the optimum point is close to an intermediate social opening, controlled by the isolation of groups of people of higher risk and opening of groups of people of lower risk.

To confirm this evidence, similar conclusions can be drawn from the recent work of Acemoglu et al [4]. The authors have studied via SIR models the effects of social isolation on the number of deaths and on the GDP reduction of the United States and have concluded that, for constant GDP reduction, there is significative reduction in the number of deaths by using vertical social isolation in comparison to any other type of isolation or lockdown. 


\section{5 - POSSIBLE EXTENSIONS}

The problem discussed in this paper may be extended to the case of multiple threats, multiple factors, with interconnected multiple restrictions, trying to reach multiple objectives with different weights. Another extension is the possibility of considering some sort of collaboration between the factors in the sharing of the resources, increasing the coupling between the problem elements. In addition, the problem can be even further generalized by making all these elements time-varying, which transforms static into dynamic scenarios.

Here we have discussed at the conception level the two-dimensional and static case, that can be visualized on a plane. With three dimensions, the solution can be sought in space. However, there is no limitation in going into the mathematical $\mathrm{N}$-dimensional space. Either in the static or dynamic case, the model will continue to represent a complex multidimensional minimization problem with one or more minima.

The discussion in this paper has been mostly qualitative, for this is a conception paper. Natural extensions should consider the modeling of factors, variables, and restrictions, in order to allow for computational simulations. Once they are handy, they can be confronted with field results for model validations and adjustments.

Some of the extensions presented here, with details, are discussed in the Annex. Other new ones are introduced.

\section{6- CONCLUSIONS}

Out of clutter, find simplicity. Albert Einstein

A model to allow macroanalysis of complex systems facing threats with competing factors has been presented. The systems are stressed by threats and their outcomes are undesirable. The central idea of this research is to find the best rational way to employ the competitive factors in order to minimize the outcomes.

A baseline problem has been established by considering the ideal case in which the competing factors have no restrictions. From the baseline problem a theory has been constructed to deal with more general problems with restrictions based on the Principle of Energy.

The mathematical model of the problem has been applied up to the first level in a chain of three levels. Despite the complexity of the scenario and the resulting model, it is shown here that the final solution is simple, as indicated by Einstein's quote.

The theory has been applied to the case of the COVID-19 virus as a threat to a country with two competitive factors: Health and Economy. Within the hypotheses and qualitative conditions established by the model, it is shown that, in order to minimize 
the total number of deaths produced by the virus, the best recommendation is to balance the emphasis on Health and Economy using the criticality of each factor as weight. This conclusion implies, for instance, that a vertical social isolation model has to be enforced to bring the system stresses to equilibrium and, in consequence, the number of deaths to a minimum.

The social isolation model is not the only possibility, for the Economy Factor $\alpha_{2}$ has several other variables and restrictions to be considered and analyzed. This is an open question, like many others created within the conception introduced in this article.

The Mathematics used to analyze problems of epidemics has been based on the solution of differential equations of the SIR model (Kermack and McKendrick [5]). In this paper a different approach has been proposed, based on the optimization of an integral objective function inspired by the quadratic form of the Energy Principle. The approach used here is more general, in the sense that it can deal with the allocation of resources and provide useful and more comprehensive information for decision-making.

The model presented here can be extended to more general cases. In addition, the model can be developed further in order to evolve from the present qualitative to a future quantitative status. Then, it may allow for computational simulation and comparison with field statistics for validation and forecasting. In this way, it may become a useful tool for authorities charged with decision-making by reducing guessing and uncertainty in complex scenarios.

\section{7 - CONFLICT OF INTEREST}

The author declares that he has no conflict of interest.

\section{8 - REFERENCES}

[1]. Gelfand, I. M.; Fomin, S. V. (2000). Silverman, Richard A. (ed.). Calculus of Variations (Unabridged repr. ed.). Mineola, New York: Dover Publications. p. 3. ISBV 9780486414485.

[2]. Goldstine, H. H. (2012). A History of the Calculus of Variations from the $17^{\text {th }}$ through the $19^{\text {th }}$ Century. Springer Science \& Business Media. p. 110. ISBN 9781461381068.

[3]. Ferguson, J. (2004). "Brief Survey of the History of the Calculus of Variations and its Applications". arXiv: math/0402357.

[4]. Acemoglu, D.; Chernozhukov, V.; Werning, I.; Whinston, M. D. (2020). "Optimal Targeted Lockdowns in a Multi-Group SIR Model". National Bureau of Economic Research Working Paper 27102. http://www.nber.org/papers/w27102.

[5]. Kermack, W. O.; McKendrick, A. G. (1927). "A Contribution to the Mathematical Theory of Epidemics". Proceedings of the Royal Society A: Mathematical, Physical and Engineering Sciences. 115 (772): 700. Bibcode:1927RSPSA.115..700K. doi:10.1098/rspa.1927.0118. JSTOR 94815. 
[6]. Yates, C. (2020). "How to Model a Pandemic". The Conversation. https://theconversation.com/how-to-model-a-pandemic-134187.

[7]. Klein, D. Lagrange Multipliers without Permanent Scarring. Computer Science Division, University of California at Berkeley. Tutorial available in https://people.eecs.berkeley.edu/ klein/papers/lagrange-multipliers.pdf. 


\section{ANNEX - THE RELEVANT MATHEMATICS}

The threat-outcome problem is discussed here in general terms. The application to an epidemic or pandemic is a case of immediate concern that has prompted this study, but this theory is not limited to this scenario. For a recent appraisal of the mathematical tools available for the study of pandemics, the reader is referred to Yates [6].

The most used mathematical approach to treat this problem has been presented in 1927 by Kermack and McKendrick [5]. It divides the domain of people threatened by an epidemic in three groups: the Susceptibles (S), the Infectives (I), and the Removed (R). It is referred to as the SIR model.

For almost a century now, the SIR model has been used as the main analysis tool of this theme. It is represented by a coupled set of three linear differential equations in time with initial conditions, which are integrated numerically by computers. This represents the state-of-the-art.

The concept presented in this paper is novel, in the sense that it is an integral, rather than a differential method. It can incorporate the dynamics of the SIR model and the allocation of resources as an advantage. Finally, it can be applied to any threat-outcome scenario, being not limited to epidemics.

Let us consider the mathematics of the general case of a single objective function with multiple competing factors. Let I represent an integral function that can be expressed as a general quadratic form of factors $\alpha_{i}(i=1,2, \ldots \mathrm{m})$. Let us suppose that I obeys a stress-relieving principle like the Principle of Energy. The factors may be expressed in terms of variables $\mathrm{x}_{\mathrm{j}}(\mathrm{j}=1,2, \ldots \mathrm{n})$ which can also be functions of time. With this enchained notation, Equation (1) in the text may be rewritten as follows:

$$
\delta I\left(\alpha_{i}\left(x_{j}(t)\right)\right)=0 \quad i=1,2, \ldots m \quad j=1,2, \ldots n
$$

\section{A.1 - The Three Analysis Levels}

In the first level of analysis, not considering the modeling details introduced by the variables $x_{j}(j=1,2, \ldots n)$, Equation (22) can be expanded as

$$
\frac{\partial \mathrm{I}}{\partial \alpha_{\mathrm{i}}} \delta \alpha_{\mathrm{i}}=0
$$

Since every variation $\delta \alpha_{i}(i=1,2 \ldots m)$ cannot be equal to zero, we end up with

$$
\frac{\partial \mathrm{I}}{\partial \alpha_{\mathrm{i}}}=0 \quad \mathrm{i}=1,2 \ldots \mathrm{m}
$$


If $I$ is indeed a quadratic form of the factors $\alpha_{i}$, Equation (24) represents a system of linear equations of order $m$, as we have shown in the application developed in section 4.3, with Equation (6). If I is not quadratic, Equation (24) represents a set of $m$ nonlinear equations that can be solved by numerical computational methods.

To second level, we have first to expand all $m$ factors $\alpha_{i}$ in terms of the $n$ variables $\mathrm{x}_{\mathbf{j}}$. Then, the problem of variation of I (Equation 22) goes like this:

$$
\frac{\partial \mathrm{I}}{\partial \alpha_{\mathrm{i}}} \frac{\partial \alpha_{\mathrm{i}}}{\partial \mathrm{x}_{\mathrm{j}}} \delta \mathrm{x}_{\mathrm{j}}=0
$$

Since every variation $\delta x_{j}(j=1,2 \ldots n)$ cannot be equal to zero, we obtain

$$
\frac{\partial I}{\partial \alpha_{i}} \frac{\partial \alpha_{i}}{\partial x_{j}}=0 \quad j=1,2 \ldots n
$$

which represents a system of linear or nonlinear coupled equations of order $\mathrm{n}$ that can be solved by numerical methods.

In the third level, we consider that all variables $x_{j}(j=1,2, \ldots n)$ are functions of time $t$. If all $\mathrm{x}_{\mathrm{j}}$ are constant with time, we have a static scenario. In the general case, the scenario has dynamics described by

$$
x_{j}=x_{j}(t) \quad(j=1,2, \ldots n)
$$

Then, equations (26) can be generalized as follows:

$$
\frac{\partial \mathrm{I}}{\partial \alpha_{\mathrm{i}}} \frac{\partial \alpha_{\mathrm{i}}}{\partial \mathrm{x}_{\mathrm{j}}} \frac{\partial \mathrm{x}_{\mathrm{j}}}{\partial \mathrm{t}}=0
$$

Now, equation (28) represents a set of $n$ coupled linear or nonlinear differential equations that can be used to describe the dynamics of the scenario. In this sense, at this point of the analysis the present approach may incorporate or establish a link with the SIR model and seek a solution with numerical integration in time.

Once a solution is obtained for each of the $n$ variables $x_{j}(t)$, the time history of each of the $m$ factors $\alpha_{i}(t)$ can be constructed by reversing the sense of analysis. From these results, it is possible to simulate all the scenario dynamics, provided there is a complete set of initial and boundary conditions to yield a well-posed mathematical problem. 


\section{A.2 - Inclusion of Restrictions}

Let us suppose that both factors $\alpha_{i}(i=1,2, \ldots m)$ and variables $x_{j}(j=1,2, \ldots n)$ may suffer dynamic restrictions $\rho_{\mathrm{k}}(\mathrm{k}=1,2, \ldots \mathrm{p})$ in the form

$$
\rho_{\mathrm{k}}=\rho_{\mathrm{k}}\left(\alpha_{\mathrm{i}}, \mathrm{x}_{\mathrm{j}}, \mathrm{t}\right) \quad \mathrm{k}=1,2, \ldots \mathrm{p}
$$

Here it is assumed that the restrictions $\rho_{k}$ are expressed in the same dimensional form of the integral objective function I. This implies that if I is energy, so must be every weighted restriction $\rho \mathrm{k}$.

The incorporation of the restrictions into I can be made through of $\mathrm{p}$ Lagrange multipliers $\lambda_{\mathrm{k}}$ (Klein [7]) that change Equation (22) to

$$
\delta\left(\mathrm{I}-\lambda_{\mathrm{k}} \rho_{\mathrm{k}}\right)=0
$$

Again, for the sake of brevity, we will present here just the first level of analysis. The variation of Equation (30) changes Equation (26) to

$$
\frac{\partial \mathrm{I}}{\partial \alpha_{\mathrm{i}}}-\lambda_{k} \frac{\partial \rho_{k}}{\partial \alpha_{\mathrm{i}}}=0 \quad \mathrm{i}=1,2, \ldots \mathrm{m} \quad \mathrm{k}=1,2, \ldots \mathrm{p}
$$

which represents a set of $\mathrm{m}$ linear or nonlinear equations, depending on the mathematical representation of I and of each $\rho_{k}$.

\section{A.3 - The Case of Multi-objective Functions and Multi-factors}

The objective function I that represents the sum of all the energy of the domain may be, in the general case, represented by the sum of two or more objective functions. For instance, the simultaneous minimization of number of deaths and of GDP reduction due to the pandemic appears in the Acemoglu et al [4] study.

Here, for the purpose of discussion, let us consider the case where the problem has two objective functions weighted by a single weight $w$, so that $0 \leq w \leq 1$, and two competing factors, so that

$$
\mathrm{I}\left(\alpha_{1}, \alpha_{2}\right)=\mathrm{w} \mathrm{I}_{1}\left(\alpha_{1}, \alpha_{2}\right)+(1-\mathrm{w}) \mathrm{I}_{2}\left(\alpha_{1}, \alpha_{2}\right)
$$


The case of a single objective function is recovered if $w$ is set exactly equal either to 0 or to 1 . In this weighted case, the first level Equation (5) is generalized to

$$
\left[w \frac{\partial I_{1}}{\partial \alpha_{i}}+(1-w) \frac{\partial I_{2}}{\partial \alpha_{i}}\right] \delta \alpha_{i}=0 \quad i=1,2
$$

which can be rewritten in extended form as

$$
\left[w \frac{\partial I_{1}}{\partial \alpha_{1}}+(1-w) \frac{\partial I_{2}}{\partial \alpha_{1}}\right] \delta \alpha_{1}+\left[w \frac{\partial I_{1}}{\partial \alpha_{2}}+(1-w) \frac{\partial I_{2}}{\partial \alpha_{2}}\right] \delta \alpha_{2}=0
$$

Since the variations of $\alpha_{\mathrm{i}}(\mathrm{i}=1,2)$ cannot be both simultaneously equal to zero, the result is the set of equations

$$
\begin{gathered}
\frac{\partial I}{\partial \alpha_{1}}=w \frac{\partial I_{1}}{\partial \alpha_{1}}+(1-w) \frac{\partial I_{2}}{\partial \alpha_{1}}=0 \\
\frac{\partial I}{\partial \alpha_{2}}=w \frac{\partial I_{1}}{\partial \alpha_{2}}+(1-w) \frac{\partial I_{2}}{\partial \alpha_{2}}=0
\end{gathered}
$$

This is a coupled set of two linear or nonlinear equations, depending on the nature of the two objective functions $I 1$ and $I_{2}$.

Another extension can consider the inclusion in the analysis of a third factor, e.g., linked to social issues. This model provides a more general and balanced representation of the real scenario humanity faces now with the COVID-19 threat. This and other themes may be explored in future studies following the integral optimization approach introduced in this paper. 
Figures

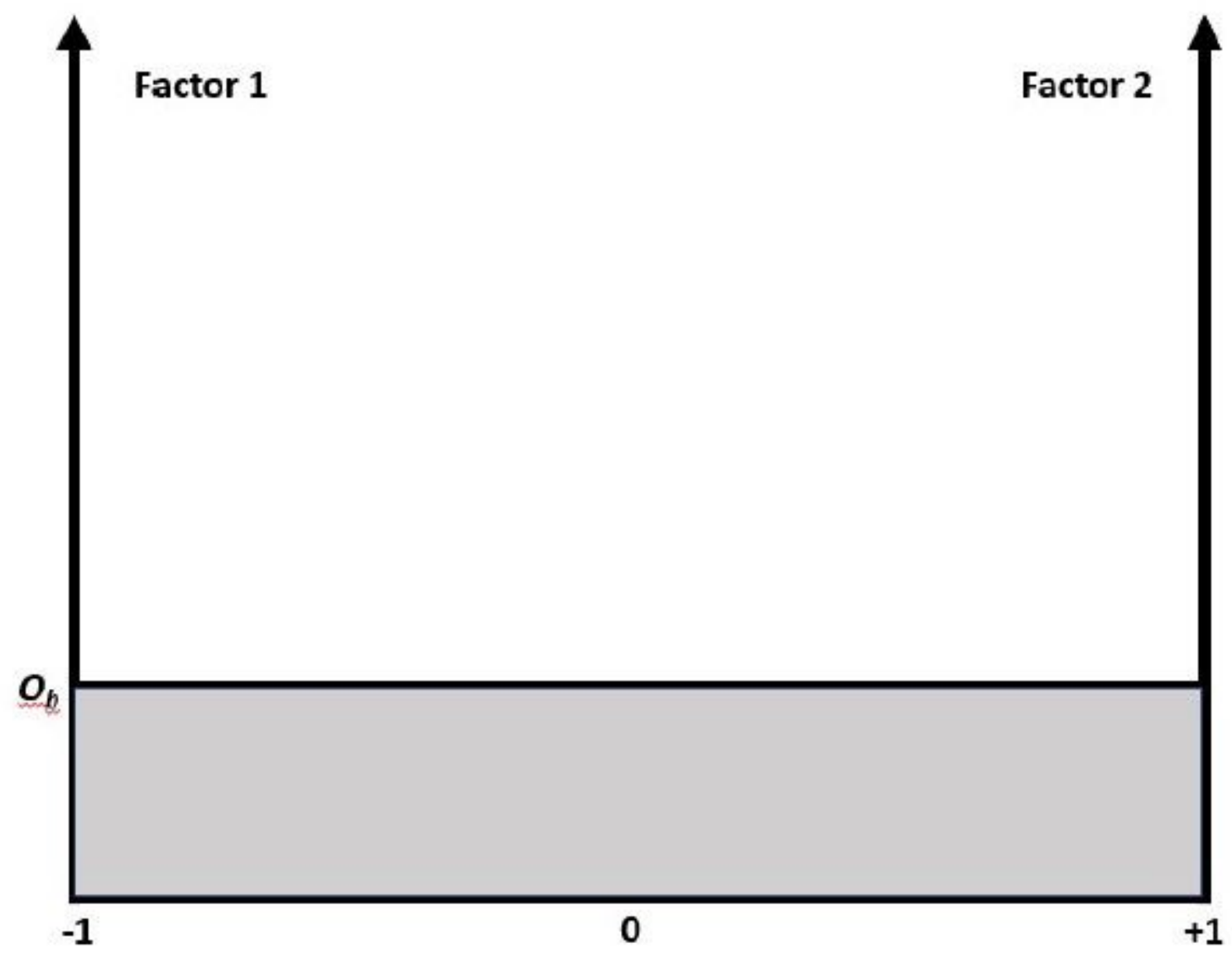

Figure 1

The baseline unrestricted problem 


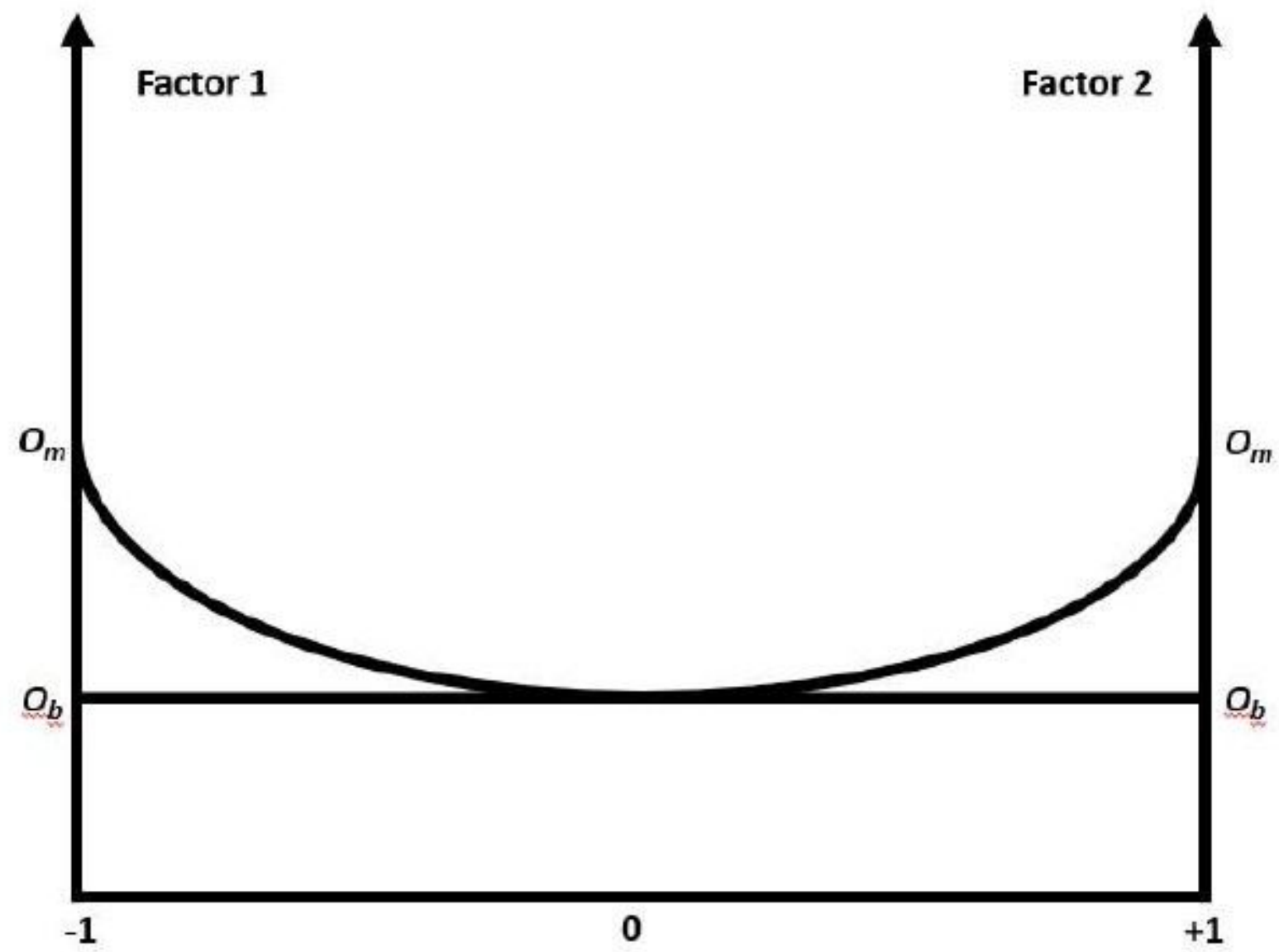

Figure 2

The perfectly symmetrical competing problem 


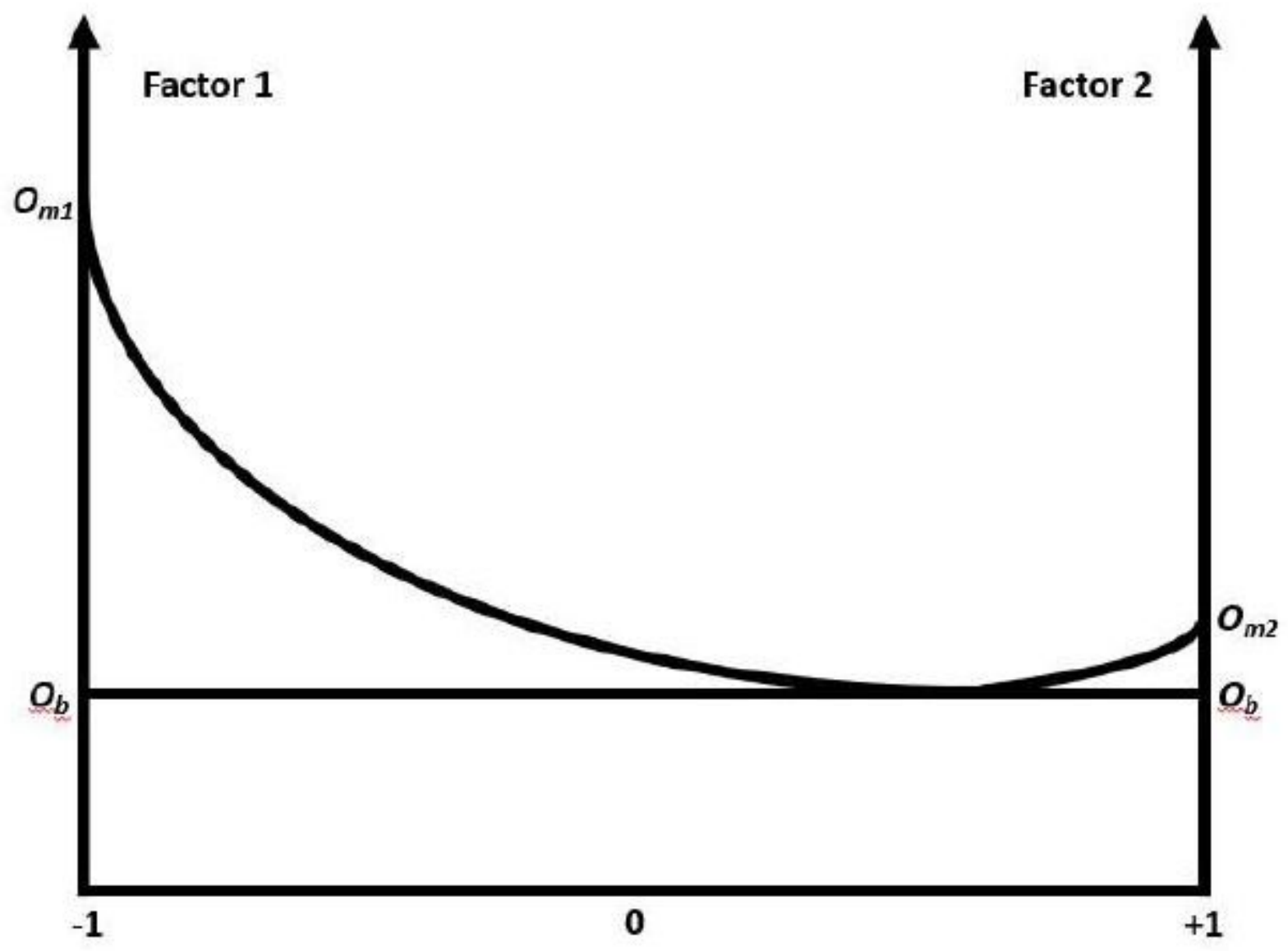

Figure 3

An asymmetrical competing problem 


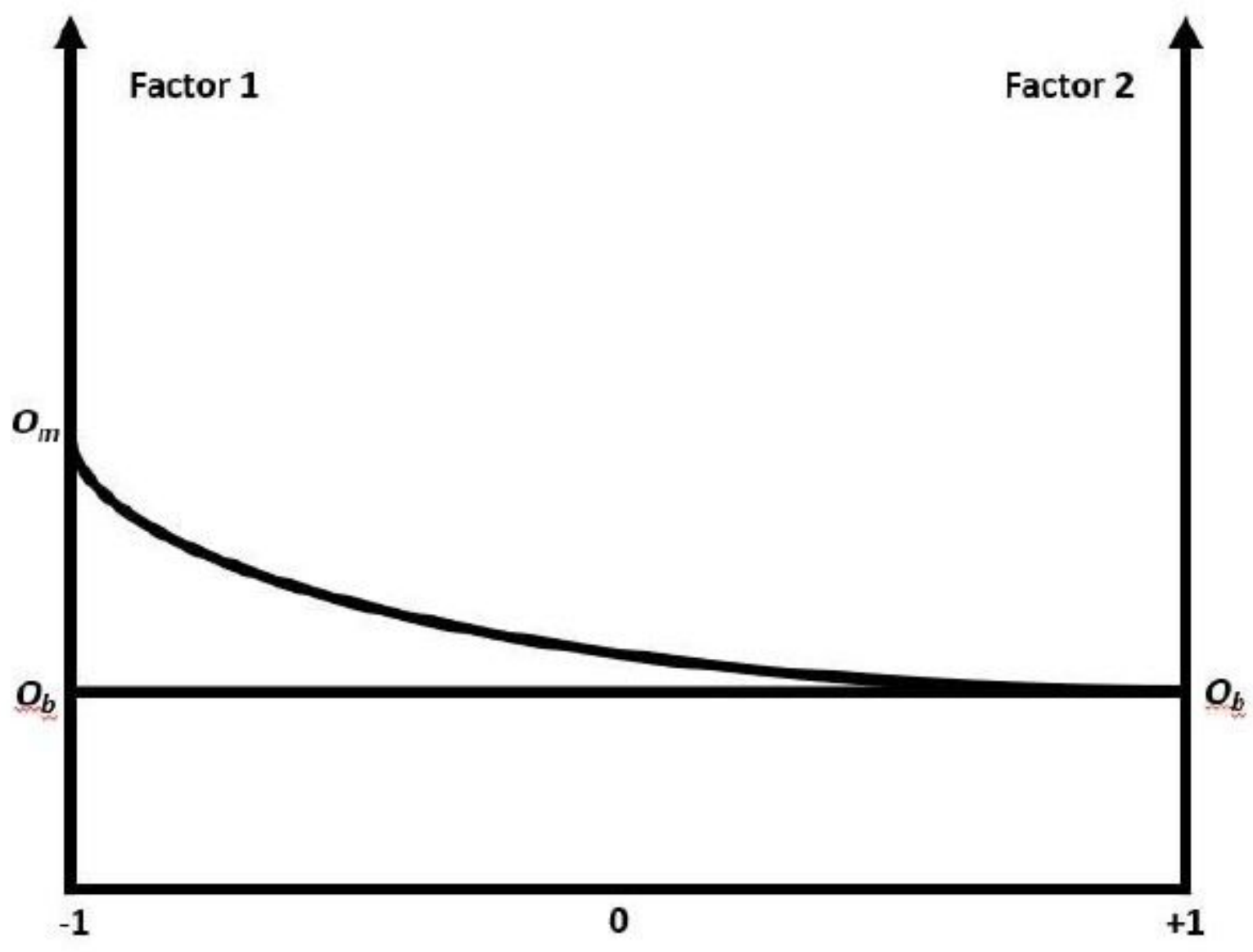

Figure 4

A limiting asymmetrical competing problem 